Virgilio Gallai

Paola Sarchielli

for the Ad Hoc Committee

for the Diagnostic and

Therapeutic Guidelines

of Migraine and Cluster

Headache

\section{Diagnostic and therapeutic guidelines for migraine. Italian Society for the Study of Headaches (SISC)}

V. Gallai $(\bowtie) \cdot$ P. Sarchielli

Department of Neuroscience,

University of Perugia,

Policlinico Monteluce,

Via E. Dal Pozzo,

I-06126 Perugia, Italy

e-mail: gallai@unipg.it

Tel. +39-075-573871

Fax: +39-075-5783583

\begin{abstract}
The Italian Society for the Study of Headaches (SISC) published the first guidelines for diagnosis and treatment of migraine in 1993. In 2000, the Executive Committee of the SISC decided to provide updated diagnostic and therapeutic guidelines for migraine and cluster headache for headache experts and physicians dedicated to headaches. For this purpose an Ad Hoc Committee was formed, taking into consideration the multidisciplinary characteristics of the society, and was composed of prominent Italian researchers from various disciplines. The guidelines were published in 2001 and will be updated each year on the basis of new information concerning diagnostic and therapeutic strategies for migraine and cluster headache. The Ad Hoc Committee was organized into subcommittees, each responsible for a different topic: diagnosis of migraine; symptomatic treatment of migraine; prophylactic treatment of migraine; non-pharmacological treatment of migraine; diagnosis, symptomatic and prophylactic treatment of cluster headache; and lastly trigeminal autonomic cranial neuralgias (TACs) and unsolved questions. The present paper reports the major conclusions of the first three subcommittees only. From a methodological point of view, it was decid-
\end{abstract}

ed that all the information reported in the guidelines would be evidencebased. This involved a thorough research on Medline and Pubmed taking into consideration all the articles concerning clinical, laboratory and instrumental examinations and therapeutic approaches for migraine and cluster headache. As far as migraine is concerned, all studies available in the literature examining the sensitivity, specificity, predictive value of clinical history, neurological examination, laboratory tests and instrumental examination were evaluated to identify variables useful in the differential diagnosis of secondary headaches mimicking migraine attacks and to identify surrogate indicators of migraine. Four groups of recommendations, based on the levels of evidence, scientific effect and clinical assessment were defined for all symptomatic and prophylactic drugs. Information regarding clinical contraindications and pharmacological interactions for each class of symptomatic and prophylactic anti-migraine drugs were also reported.

Key words Guidelines • Italian Society for the Study of Headaches • Migraine - Diagnosis • Treatment • Symptomatic drugs • Prophylactic drugs $\cdot$ Recommendations 


\section{Introduction}

The Italian Society for the Study of Headaches (SISC) was the first scientific society to publish, in 1993, diagnostic and therapeutic guidelines for migraine [1]. These guidelines represented a milestone in the history of SISC. In the last few years, the need to provide an updated diagnostic and therapeutic orientation for headache experts and physicians dedicated to headache was pressing for the society, not only because of the remarkable progress made in the treatment strategies for headaches, but also because of the publication of headache guidelines by the scientific societies of other countries (e.g. Canada, Denmark, England, U.S.A.) [2-7].

The Ad Hoc Committee for the Diagnostic and Therapeutic Guidelines for Migraine and Cluster Headache was formed in consideration of the multidisciplinary characteristics of the society and was composed of prominent Italian researchers from various disciplines. In particular, committee members were selected from the membership of the SISC, the Society of Neurology, the Society of Childhood and Adolescence Neuropsychiatry, the Society of Internal Medicine, the Society of Pain Clinicians, the Society of Clinical Pharmacology and the Society of Emergency Physicians. Additionally, two headache patients were invited to participate in the committee. The Ad Hoc Committee was organized into subcommittees, each responsible for a different topic: diagnosis of migraine; symptomatic treatment of migraine; prophylactic treatment of migraine; non-pharmacological treatment of migraine; diagnosis, symptomatic and prophylactic treatment of cluster headache; and lastly trigeminal autonomic cranial neuralgias (TACs) and unsolved questions. The present paper reports the major conclusions of the first three subcommittees only.

The initial activity of the Ad Hoc Committee consisted in establishing precise, practical guidelines to be used by specialists and general practitioners. The guidelines in this manner will be validated directly in the field and must therefore be regularly revised based on the new information acquired.

From a methodological point of view, it was decided that all the information reported in the guidelines would be evidence-based [8]. Keeping this in mind, the subcommittees carried out a thorough research in Medline and Pubmed, taking into consideration all the articles concerning clinical, laboratory and instrumental examinations and therapeutical approaches. Abstracts were excluded. The subcommittees then proceeded to assess these studies by attributing a score in decreasing value to: controlled, randomised, doubleblind, placebo studies, carried out according to good clinical practice (GCP); meta-analyses; followed by prospective and transversal studies, reviews, case reports, and lastly anecdotic experiences [9].
Statistical assessment was then used, and involved taking the results of these studies and establishing the strength of the evidence.

Lastly, the members of each subcommittee, based on their personal clinical experience, gave the results of the studies a clinical evaluation.

\section{Diagnosis of migraine}

All members of the subcommittee agreed that the diagnosis of migraine should be based exclusively on the current diagnostic criteria of the International Headache Society (IHS). The need for a clinical diagnosis derives from the lack of specific biological markers for the definition of migraine and the incompleteness of the knowledge of the pathophysiological mechanisms underlying this primary headache.

All studies available in the literature examining the sensitivity, specificity, predictive value of clinical history, neurological examination, laboratory tests and instrumental examination were evaluated with the final objective of defining the variables to be used in differentiating diagnosis for secondary headaches mimicking migraine attacks and to identify surrogate indicators of migraine. On the basis of evidence found in literature, unanimous consensus was reached among the members that it is necessary to collect clinical history, and to perform general and neurological examinations, with the aim of identifying "hot spots" which may indicate a secondary headache. In this regard, it is important to remember that some disorders underlying headache are considered "dangerous", and that a few of them may put the life of the patient at risk.

The following anamnestic information may suggest a secondary headache disorder:

1. A variation in the severity of crises. Particular attention should be dedicated to crises which are defined by the same patient as "being the worst in their life".

2. A variation in the characteristics of headache leads to suspect a secondary headache.

3. A variation in the frequency of crises, especially if the frequency is rapidly increasing.

4. A progressive course of headache always suggests a secondary headache.

5. Frequent awakening during the night with headache may suggest a secondary headache with migraine-like characteristics, although in some cases migraine, as a primary disorder, may also occur during the night.

6. A headache which worsens after the Valsalva manoeuvre (cough, sneeze or flexion of the head) should be further investigated.

7. Association with systemic signs. The presence of myalgia, fever or weight loss may indicate the presence of a secondary headache. 
8. Association with other neurological signs. The association with state of confusion, alteration of alertness, loss of consciousness, or epileptic crises suggests a secondary headache.

9. Resistance to both symptomatic and prophylactic antimigraine drugs.

10. Age at onset greater than 40 years.

11. A recent onset.

A general examination (measurement of blood pressure, heart rate and body temperature; examination of paranasal sinuses, carotid arteries and cervical paravertebral muscles; check for alterations in the mandibular-temporal articulations, etc.) is mandatory to exclude secondary headaches. A neurological examination should always be carried out to exclude neurological alterations. Neuroimaging is not a routine procedure, but should be carried out only in: (a) patients with neurological signs; (b) patients with negative neurological examination but with neurological symptoms; and (c) patients with a headache that changes characteristics or presents other characteristics indicated in the list of anamnestic information suggesting a secondary headache. Brain magnetic resonance imaging (MRI) has greater sensitivity than computed tomography $(\mathrm{CT})$ in revealing white matter abnormalities, arteriovenous malformations and lesions in the posterior cranial fossa. The choice between the two examinations is based on the clinical suspicion of the physician.

Electroencephalography (EEG) is not useful in the routine diagnostic evaluation of patients suffering from migraine and more generally from headache. However, EEG may be recommended for patients with headache and symptoms suggesting epileptic crises (which can be atypical migraine aura, loss of consciousness, etc.).

There is no experimental evidence showing that further laboratory tests carried out on blood or cerebrospinal fluid can demonstrate sensitive and specific alterations from a diagnostic point of view. This is also true for neurophysiological, radiological and histological examinations, as well as ultrasonography, which should be requested, on the basis of clinical suspicion, to exclude the possibility of a secondary headache.

Definition of the level of evidence, scientific effect and clinical assessment

On the basis of studies in the literature, the levels of evidence for each symptomatic and prophylactic antimigraine drug and also the scientific strength of the evidence have been defined. The clinical assessment was also given for each drug on the basis of the consensus reached between the members of Ad Hoc Committee. As far as the strength of evidence is concerned, 3 classes were established in evaluating the studies: Class A, 2 or more clinically controlled, randomised studies carried out according to the GCP, versus placebo or versus active treatment of proven efficacy; Class $B, 1$ clinically controlled, randomised study carried out according to the GCP or more than one well-designed clinical case-control study or cohort study; Class $C$, favourable judgement of two-thirds of the Ad Hoc Committee members, historical controls, non-randomised studies or case reports.

The level of statistical significance and the strength of clinical response of the evidence was defined by the Ad Hoc Committee in a more precise manner compared to the American guidelines for migraine. The members, with the aim of avoiding the generic terms "most" and "few" used in the definition of the clinical impression in the American guidelines [6], decided that the clinical assessment would be expressed in percentages based on clinical patient benefit. Clinical assessment was attributed 4 levels $(+++,++,+, 0)$. Four groups of recommendations, based on the levels of evidence, scientific effect and clinical assessment were defined for symptomatic and prophylactic drugs:

I. Drugs with high efficacy supported by statistically significant data (evidence of at least two controlled, randomized studies versus placebo or versus active drugs of proven efficacy) or high clinical benefit for patients (clinical assessment +++) and with infrequent adverse events.

II. Drugs whose value of efficacy is statistically of lower significance compared to drugs of Group I and with a less significant clinical benefit for patients (clinical assessment ++ ) and slight or moderate adverse events.

III. Drugs showing efficacy from a statistical point of view but not from a clinical point of view (contrasting results or inconclusive evidence). The drugs belonging to this group were further subdivided in two subgroups:

a. Drugs with adverse events of slight or moderate intensity;

b. Drugs with uncertain safety or with complex indications for use (e.g. special diets) or important pharmacological interactions.

IV. Drugs of proven efficacy but with frequent and severe adverse events or drugs whose efficacy has not been proven from a clinical or statistical point of view (no difference with respect to placebo). Drugs with unknown clinical patient benefit or statistical significance of efficacy (data not available or insufficient).

\section{Symptomatic treatment of migraine}

Drugs included under the recommendations of Group I are: all triptans, some analgesics and non-steroidal anti-inflammatory drugs (NSAIDs, e.g. acetylsalicylic acid, lysine sal- 
icylate, ibuprofene, sodium naproxene) and dihydroergotamine nasal spray. Consistent data support their efficacy and tolerability.

Group II includes diclofenac per os, ketoprofene, ketorolac, naproxen, acetaminophen, some antiemetics administered intravenously (prochlorperazine, chlorpromazine, metoclopramide), ergotamine administered intramuscularly and subcutaneously, and finally dihydroergotamine administered orally, subcutaneously, intramuscularly and intravenously (the latter is not available in Italy).

The drugs included under Group III are: the combination butalbital+acetylsalicylic acid+caffeine; oral ergotamine, with or without caffeine; indomethacin+prochlorperazine+caffeine combination; intravenous lidocaine, which shows a modest efficacy but is associated with high risk of headache recurrence and, among the NSAIDs, the rapid dissolving form of piroxicam.

The following drugs were included in Group IV because of a limited or a lack of scientific evidence on efficacy: intravenous dexamethasone and hydrocortisone, for which there is little and inconclusive clinical experience supporting the efficacy in the migrainous status; and intravenous granisetron and oral metoclopramide, both considered exclusively as adjuvants in the treatment of attacks when vomiting prevails. Group IV also includes nimesulide, for which there is no evidence of efficacy in the symptomatic treatment of migraine attacks and few data supporting its efficacy in the intermittent treatment of menstrual migraine. The use of analgesic combinations is not recommended by the Ad Hoc Committee due to the high risk of abuse and chronicization of headache. This risk, together with the risk of dependence, was also stated for drugs containing barbiturates and narcotics. In particular, the Committee unanimously agreed to exclude the use of analgesic opioids in the treatment of migraine attacks.

\section{Prophylactic treatment of migraine}

The same criteria used for the assessment of the efficacy of symptomatic drugs were adopted for prophylactic treatment of migraine. The conclusions reached by the Ad Hoc Committee regarding certain prophylactic drugs differ, at least in part, from those reached by the American Consortium.

The present guidelines include few prophylactic drugs under the recommendations of Group I on the basis of evidence, scientific effect and clinical assessment. They are: amitriptyline, atenolol, propranolol, flunarizine, and val- proate sodium. Group II includes: the NSAIDs, specifically naproxen and naproxen sodium (the use of which is limited to the intermittent prophylaxis of menstrual migraine) and also lornoxicam; the antiepileptic drugs gabapentin, lamotrigine, topiramate; the calcium channel blockers verapamil and cinnarizine; the selective serotonin reuptake inhibitor (SSRI) fluoxetine; the beta-blockers metoprolol and nadolol; dihydroergotamine and vitamin B2.

The following drugs were classified under Group III subgroup a: nortriptiline, which is less efficient but is better tolerated than amitriptyline; the SSRIs fluvoxamine, paroxetine and sertraline; magnesium, lisuride, diltiazem and finally botulin A toxin. Acetylsalicylic acid and pizotifen were included in Group III subgroup b. Other drugs whose evidence of efficacy are lacking, limited or contrasting (Group IV recommendation) are: buspirone, carbamazepine, clonidine, dotiepine, estradiol, imipramine, ketoprofen mertazepine, trazodone, venlafaxine and, finally feverfew. Lastly, all members of the Ad Hoc Committee agreed to not recommend monoamine oxidase inhibitors for the prophylactic treatment of migraine. The first edition of the Guidelines for the diagnosis and treatment of migraine and cluster headache has been published in the Italian SISC journal (June, 2001) [10].

The Ad Hoc Committee for the Diagnostic and Therapeutic Guidelines of Migraine and Cluster Headache is composed of: Virgilio Gallai (President of SISC and coordinator, Symptomatic Treatment of Migraine Subcommittee), Andrea Alberti, Massimo Alessandri, Luigi Arturo Ambrosio, Vincenzo Bonavita, Ubaldo Bonuccelli, Gennaro Bussone, Gabriella Maria Buzzi, Rosolino Camarda, Franca Carboni, Cesare Colucci D'Amato, Francesco Coraggio, Claudio Cricelli, Giovanni D'Andrea (Coordinator, Trigeminal Autonomic Cranial Neuralgias and Unsolved Questions Subcommittee), Milena De Marinis, Maria Del Zompo, Raoul Di Perri, Girolamo Di Trapani, Alessandro Ducati, Marcello Fanciullacci (Coordinator, Diagnosis, Symptomatic and Prophylactic Treatment of Cluster Headache Subcommittee), Sergio Genco, Mario Giacovazzo, Mario Guazzelli, Vincenzo Guidetti, Massimo Leandri, Massimo Leone (Coordinator, Nonpharmacological Treatment of Migraine Subcommittee) Gian Camillo Manzoni, Paolo Martelletti, Giovanni Mazzotta, Ubaldo Mengozzi, Sergio Michelacci, Giuseppe Nappi, Maria Carola Narbone, Biagio Panascia, Francesco Perini, Luigi Alberto Pini (coordinator, Prophylactic Treatment of Migraine Subcommittee), Francesco Pisani, Maria Pia Prudenzano, Franco Michele Puca (coordinator, Diagnosis of Migraine Subcommittee), Barbara Radaelli (headache patient), Sergio Rassu, Giovanni Regesta, Giuliano Relja, Giorgio Sandrini, Paola Sarchielli, Mariantonietta Savarese, Lidia Savi, Pier Liugi Scapicchio, Emilio Sternieri, Marco Visentin, Giorgio Zanchin 


\section{References}

1. - (1993) Guidelines and recommendations for the treatment of migraine. Italian Society for the Study of Headache (SISC). Funct Neurol 8(6):441-446

2. Capobianco DJ, Cheshire WP, Campbell JK (1996) Subspeciality clinics: Neurology. An overview of the diagnosis and pharmacologic treatment of migraine. Mayo Clin Proc 71:1055-1066

3. Pryse-Phillips WE, Dodick DW, Edmeads JG et al (1997) Guidelines for the diagnosis and management of migraine in clinical practice. Canadian Headache Society. CMAJ 156:1273-287

4. - (1998) Guidelines for the management of headaches. Danish Neurological Society and The Danish Headache Society. Cephalalgia 18:9-22
5. Diener H-C, Kaube H, Limmrothv (1998) A practical guide to the management and prevention of migraine. Drugs 56(5):811-824

6. US Headache Consortium, Headache Guidelines (April 24, 2000).

http://www.ashnet.org/guidelines.php (page viewed on 6 August 2001)

7. Steiner TJ, MacGregor EA, Davies PTG for the British Association the Study of Headache (2000) BASH Management Guidelines. Guidelines for all doctors in the diagnosis and management of migraine and tension-type headache, 2nd edn. BASH, Derbyshire (http://www.bash.org.uk/guidelines.ht, page viewed on 23 July 2001)
8. Becker WJ (2000) The challenge of evidence-based migraine therapy. $\mathrm{Ce}$ phalalgia 20[Suppl 2]:1-4

9. Tfelt-Hansen P, Block C, Diener HC, Ferrari MD, Goadsby PJ, Guidetti V, Jones B, Lipton RB, Massiou H, Meinert C, Sandrini G, Steiner T, Winner PB and The International Clinical Trial Subcommittee (2000) Guidelines for controlled trials of drugs in migraine, $2^{\text {nd }}$ edn. Cephalalgia 20 (9):765-786

10. Linee guida per la diagnosi e la terapia dell'emicrania e della cefalea a grappolo. Il Giornale SISC 1[Suppl]:1-67 